PROCEEDINGS OF THE

AMERICAN MATHEMATICAL SOCIETY

Volume 131, Number 8, Pages 2371-2377

S 0002-9939(03)06999-5

Article electronically published on March 11, 2003

\title{
A FIXED-POINT THEOREM FOR ASYMPTOTICALLY CONTRACTIVE MAPPINGS
}

\author{
JEAN-PAUL PENOT
}

(Communicated by Jonathan M. Borwein)

\begin{abstract}
We present fixed point theorems for a nonexpansive mapping from a closed convex subset of a uniformly convex Banach space into itself under some asymptotic contraction assumptions. They generalize results valid for bounded convex sets or asymptotically compact sets.
\end{abstract}

In this note we generalize a famous result by Browder [3], Göhde [6] and Kirk [8], recently extended by Luc in [14, by using the notion of an asymptotically compact subset of a Banach space. Here no compactness assumption is involved. Instead we use uniform asymptotic concepts introduced in [19] and the following definition which differs from the one in [14]; a comparison will be made later on. Note that the meaning of the word "asymptotic" is not related with the iterations of the map as in [8], [9, [10], 21] but bears on the behavior of the map at infinity.

Definition 1. Let $C$ be a subset of a Banach space $X$. We say that a map $f: C \rightarrow$ $X$ is asymptotically contractive on $C$ if there exists $x_{0} \in C$ such that

$$
\limsup _{x \in C,\|x\| \rightarrow \infty} \frac{\left\|f(x)-f\left(x_{0}\right)\right\|}{\left\|x-x_{0}\right\|}<1 .
$$

It is easy to see that this condition is independent of the choice of $x_{0} \in C$; more precisely, when it is satisfied for some $x_{0}$, then it is satisfied for any $x_{1} \in C$. The notion of an asymptotically contractive map enables us to extend to unbounded sets the results of [3], 6], 8 valid for nonexpansive self-mappings on closed convex bounded subsets of uniformly convex Banach spaces. Recall that a mapping $f$ : $C \rightarrow X$ is nonexpansive if $\left\|f(x)-f\left(x^{\prime}\right)\right\| \leq\left\|x-x^{\prime}\right\|$ for any $x, x^{\prime}$ in $C$. It is said to be demi-closed if its graph is sequentially closed in the product of the weak topology on $C$ with the norm topology on $X$.

Proposition 2. Let $X$ be a reflexive Banach space and let $C$ be a closed convex subset of $X$. Let $f: C \rightarrow X$ be a nonexpansive map which is asymptotically contractive on $C$ and such that $f(C) \subset C$ and $I-f$ is demi-closed. Then $f$ has a fixed point.

Received by the editors February 19, 2002.

2000 Mathematics Subject Classification. Primary 47H10, 47H09, 54H25, 55M20.

Key words and phrases. Asymptotic, asymptotic cone, contraction, derivative at infinity, firm asymptotic cone, fixed point, nonexpansive map. 
Proof. Let $\left(t_{n}\right)$ be a sequence of $(0,1)$ with limit 0 and let $x_{0} \in C$. For $n \in \mathbb{N}$, let $f_{n}: C \rightarrow X$ be given by

$$
f_{n}(x):=\left(1-t_{n}\right) f(x)+t_{n} x_{0},
$$

so that $f_{n}(x) \in C$ for each $x \in C$ by convexity of $C$. Since $f$ is nonexpansive, $f_{n}$ is a contraction with rate $\left(1-t_{n}\right)$. The Picard-Banach theorem ensures that $f_{n}$ has a fixed point $x_{n} \in C$. Let us show that the sequence $\left(x_{n}\right)$ is bounded. If this is not the case, taking a subsequence if necessary, we may assume that $\left(\left\|x_{n}\right\|\right) \rightarrow \infty$. Let $\alpha \in(0,1)$ and $\rho>0$ be such that $\left\|f(x)-f\left(x_{0}\right)\right\| \leq \alpha\left\|x-x_{0}\right\|$ for $x \in C$ satisfying $\|x\| \geq \rho$. Then, for $n$ large enough, we have

$$
\begin{aligned}
\left\|x_{n}\right\| & =\left\|\left(1-t_{n}\right) f\left(x_{n}\right)+t_{n} x_{0}\right\| \\
& \leq\left(1-t_{n}\right)\left(\alpha\left\|x_{n}-x_{0}\right\|+\left\|f\left(x_{0}\right)\right\|\right)+t_{n}\left\|x_{0}\right\| .
\end{aligned}
$$

Dividing both sides by $\left\|x_{n}\right\|$ and taking limits, we get $1 \leq \alpha$, a contradiction. Thus, $\left(x_{n}\right)$ and $\left(f\left(x_{n}\right)\right)$ are bounded, and $\left(\left\|x_{n}-f\left(x_{n}\right)\right\|\right)=\left(t_{n}\left\|x_{0}-f\left(x_{n}\right)\right\|\right) \rightarrow 0$.

Since $X$ is reflexive, taking a subsequence if necessary, we may assume that $\left(x_{n}\right)$ has a weak limit $\bar{x}$. Since $I-f$ is demi-closed, we get that $\bar{x}-f(\bar{x})=0$.

Corollary 3. Let $X$ be a uniformly convex Banach space and let $C$ be a closed convex subset of $X$. Let $f: C \rightarrow X$ be a nonexpansive map which is asymptotically contractive on $C$ and such that $f(C) \subset C$. Then $f$ has a fixed point.

Proof. The result is a consequence of the fact that when $X$ is uniformly convex and $f$ is nonexpansive, the map $I-f$ is demi-closed (see [4, Theorem 8.4], 24. Proposition 10.9] for instance). Note that in these references the closed convex set $C$ is assumed to be bounded, but this assumption can be avoided since the definition of demi-closedness only involves bounded sequences.

Remarks. a) In the preceding statement, one can add that the set of fixed points is closed, convex and bounded. The first two properties are proved in the usual way; the boundedness property follows immediately from (1).

b) One can deduce the preceding proposition from the classical result of [3], [6] and [8] (see also [2], [7], [22]) by applying it to a sufficiently large ball. This direct way can be deduced from the preceding proof. It also follows from the observation that $f$ is asymptotically contractive iff there exists some $c \in(0,1)$ and $r>0$ such that

$$
\|f(x)\| \leq c\|x\| \quad \forall x \in C \backslash r B_{X},
$$

where $B_{X}$ is the closed unit ball of $X$ : since $f$ is supposed to be nonexpansive, there exists $s \geq r$ such that $f\left(C \cap r B_{X}\right)$ is contained in the ball $s B_{X}$ and then $f\left(C \cap s B_{X}\right)$ is contained in $C \cap s B_{X}$.

Let us compare the preceding result with [14, Theorem 5.1]. There, $C$ is supposed to be asymptotically compact in the sense of [1, 15], [18, 20] (see also [5], [17, 23]): for any sequence $\left(x_{n}\right)$ of $C$ such that $\left(r_{n}\right):=\left(\left\|x_{n}\right\|\right) \rightarrow \infty$, the sequence $\left(r_{n}^{-1} x_{n}\right)$ has a converging subsequence. This assumption is obviously satisfied in finite dimensions; it is rather restrictive in infinite-dimensional spaces. However, locally compact convex sets and epigraphs of hyper-coercive functions (i.e. functions $f$ such that $f(x) /\|x\| \rightarrow+\infty$ as $\|x\| \rightarrow+\infty$ ) are asymptotically compact (see [20]).

On the other hand, the asymptotic condition imposed on $f$ in [14] is milder than the one considered here: $f$ is just supposed to be radially asymptotically contractive 
in the sense that for some $x_{0} \in C$ and for any $u$ in the asymptotic cone (or horizon cone)

$$
C_{\infty}:=\lim \sup _{t \rightarrow \infty} t^{-1} C:=\left\{v \in X: \exists\left(t_{n}\right) \rightarrow+\infty,\left(v_{n}\right) \rightarrow v, t_{n} v_{n} \in C \forall n\right\}
$$

of $C$ one has

$$
\limsup _{t \rightarrow \infty, x_{0}+t u \in C} \frac{1}{t}\left\|f\left(x_{0}+t u\right)-f\left(x_{0}\right)\right\|<1 .
$$

This condition is obviously satisfied if $f$ is asymptotically contractive on $C$. In fact, if $f$ is nonexpansive, or even Lipschitzian, $f$ is radially asymptotically contractive if, and only if, it is directionally asymptotically contractive in the sense that for any $u \in C_{\infty}$ one has

$$
\limsup _{t \rightarrow \infty, v \rightarrow u, x_{0}+t v \in C} \frac{1}{t}\left\|f\left(x_{0}+t u\right)-f\left(x_{0}\right)\right\|<1
$$

and one has the following relationships with our assumption.

Lemma 4. Any asymptotic contraction $f: C \rightarrow X$ is a directional asymptotic contraction. If $C$ is asymptotically compact the converse holds.

Proof. The first assertion is immediate. Let us prove the second one. Let us assume that $f$ is not an asymptotic contraction: for any $x_{0} \in C$ there exists a sequence $\left(x_{n}\right)$ in $C$ such that $\left(\left\|x_{n}\right\|\right) \rightarrow \infty$ and $\lim _{n} t_{n}^{-1}\left\|f\left(x_{n}\right)-f\left(x_{0}\right)\right\| \geq 1$ for $t_{n}:=$ $\left\|x_{n}-x_{0}\right\|$. Since $C$ is asymptotically compact, the sequence $\left(u_{n}\right):=\left(t_{n}^{-1}\left(x_{n}-x_{0}\right)\right)$ has a converging subsequence. The limit $u$ of this subsequence belongs to $C_{\infty}$ and since $\lim _{n} t_{n}^{-1}\left\|f\left(x_{0}+t_{n} u_{n}\right)-f\left(x_{0}\right)\right\| \geq 1, f$ is not a directional asymptotic contraction.

It follows that [14, Theorem 5.1] is a consequence of Corollary 3,

Corollary 5 ([14]). Let $X$ be a uniformly convex Banach space and let $C$ be an asymptotically compact closed convex subset of $X$. Let $f: C \rightarrow X$ be a nonexpansive map which is radially asymptotically contractive on $C$ and such that $f(C) \subset C$. Then $f$ has a fixed point.

Let us present a criterion in order that $f$ be asymptotically contractive. It relies on the following notion introduced in [19].

Definition 6. A cone $K$ of a normed vector space $X$ is a firm (outer) asymptotic cone of a subset $C$ of $X$ if for any $\varepsilon>0$ there exists some $r>0$ such that for any $x \in C \backslash r B_{X}$ one has $d(x, K)<\varepsilon\|x\|$.

Equivalently, $K$ is a firm asymptotic cone of $C$ iff there exists some function $\alpha: X \rightarrow \mathbb{R}_{+}$such that $\alpha(x) /\|x\| \rightarrow 0$ as $\|x\| \rightarrow \infty$ for which $C$ is included in $\{x \in X: d(x, K) \leq \alpha(x)\}$. When $K$ is closed, this condition implies the inclusion $C_{\infty} \subset K$. It is easy to give examples in which this condition is satisfied and to exhibit counterexamples in which it is not satisfied, although $K$ contains the asymptotic cone $C_{\infty}$ of $C$. For instance, when $X=W \times \mathbb{R}, C:=\{(w, r) \in X: r \geq f(w)\}$, $K:=\{(w, r) \in X: r \geq h(w)\}$ for some functions $f, h: W \rightarrow \mathbb{R}$ with $h$ positively homogeneous and lower semicontinuous, $K$ is a firm asymptotic cone of $C$ whenever $\liminf _{\|x\| \rightarrow \infty}(f(x)-h(x)) /\|x\| \geq 0$. In such a case, $C_{\infty}=\{(w, r) \in X: r \geq$ $\left.f_{\infty}(w)\right\}$, where $f_{\infty}$ is the asymptotic function $f_{\infty}$ of $f$ defined by

$$
f_{\infty}(x):=\liminf _{\left(t, x^{\prime}\right) \rightarrow(+\infty, x)} \frac{1}{t} f\left(t x^{\prime}\right)
$$


and the inclusion $C_{\infty} \subset K$ amounts to $f_{\infty} \geq h$, a weaker condition. The whole space is clearly a firm asymptotic cone of any subset; one is interested in taking a cone as small as possible. The following result describes significant facts in this respect.

Lemma 7 ([19]). If $K$ is a closed firm asymptotic cone of $C$, then $K$ contains the asymptotic cone $C_{\infty}$ of $C$. If $C$ is asymptotically compact, the asymptotic cone of $C$ is a firm asymptotic cone of $C$. In particular, if $X$ is finite dimensional, for any subset $C$ of $X$ the asymptotic cone $C_{\infty}$ of $C$ is a firm asymptotic cone of $C$.

We also need a variant of a concept due to Krasnoselski [12].

Definition 8. Given another normed vector space $Y$ and a firm asymptotic cone $K$ of a subset $C$ of $X$, a positively homogeneous mapping $h: K \rightarrow Y$ is said to be a firm (outer) asymptotic semi-derivative of a mapping $f: C \rightarrow Y$ if for any $\varepsilon>0$ there exists $\rho>0$ such that for any $x \in C \backslash \rho B_{X}$ there exists $v \in K$ satisfying $\|x-v\|<\varepsilon\|x\|$,

$$
\|f(x)-h(v)\|<\varepsilon\|x\| .
$$

This condition is obviously satisfied with $h=\ell$ when $f$ has a firm (or strong) asymptotic derivative (or F-derivative at infinity) $\ell$ in the sense that $K=X$ and there exists a continuous linear mapping $\ell: X \rightarrow Y$ such that

$$
\lim _{r \rightarrow \infty} \sup _{x \in C \backslash r B_{X}} \frac{1}{\|x\|}\|f(x)-\ell(x)\|=0 .
$$

Definition 8 takes into account the fact that $f$ and $h$ are not defined on the same sets. Note that when $h: K \rightarrow Y$ is a firm (outer) asymptotic semi-derivative of $f: C \rightarrow Y$, then the graph of $h$ is a firm asymptotic cone of the graph of $f$. The converse holds when $\lim \sup _{\|x\| \rightarrow \infty}\|f(x)\| /\|x\|<+\infty$.

Example. Let $X=Y=\mathbb{R}$ and $a \in \mathbb{R}$, and let $f$ be given by $f(x):=a|x|+\sin x$. Then $h$ given by $h(x)=a|x|$ is a firm (outer) asymptotic semi-derivative of $f$.

Example. Let $f: X \rightarrow Y$ be given by $f(x):=p(a+x)$ for some given $a \in X$ and some Lipschitzian positively homogeneous map $p: X \rightarrow Y$. Then $p$ is a firm (outer) asymptotic semi-derivative of $f$.

As observed by the referee, in both examples $f$ is of the form $f=p+b$, where $b: X \rightarrow Y$ is such that $b(x) /\|x\| \rightarrow 0$ as $\|x\| \rightarrow+\infty$. Moreover, for nonexpansive maps $p$ and $b$ and $c \in(0,1)$, the function $f$ given by $f(x):=c p(x)+(1-c) b(x)$ is nonexpansive and its asymptotic semi-derivative is contractive.

The following criterion is established in [12] 3.2.2].

Lemma 9. Suppose $f: X \rightarrow X$ is Gâteaux differentiable on $X \backslash r B_{X}$ for some $r>0$ and there exists a continuous linear mapping $A: X \rightarrow X$ such that $\left\|f^{\prime}(x)-A\right\| \rightarrow 0$ as $\|x\| \rightarrow \infty$. Then $f$ has a firm asymptotic derivative $A$.

A weaker condition consists in requiring that $f: C \rightarrow X$ is asymptotable in the sense that there exists a positively homogeneous map $f_{\infty}: C_{\infty} \rightarrow X$ such that for any $u \in C_{\infty}$ one has $t^{-1} f(t v) \rightarrow f_{\infty}(u)$ as $(t, v) \rightarrow(\infty, u)$ with $t v \in C$.

Lemma 10. If $f: C \rightarrow X$ is asymptotable and if $C$ is asymptotically compact, then $f_{\infty}$ is a firm asymptotic semi-derivative of $f$. 
Proof. Suppose on the contrary that there exist some $\varepsilon>0$ and a sequence $\left(x_{n}\right)$ in $C$ with $\left(r_{n}\right):=\left(\left\|x_{n}\right\|\right) \rightarrow \infty$ such that for any $v \in C_{\infty} \cap\left(x_{n}+\varepsilon\left\|x_{n}\right\| B_{X}\right)$ one has $\left\|f\left(x_{n}\right)-f_{\infty}(v)\right\| \geq \varepsilon\left\|x_{n}\right\|$. Since $C$ is asymptotically compact, taking a subsequence if necessary, we may assume that $\left(u_{n}\right):=\left(r_{n}^{-1} x_{n}\right)$ has a limit $u$. Then $u \in C_{\infty}$ and, since $f$ is asymptotable, we have that $\left(r_{n}^{-1} f\left(r_{n} u_{n}\right)\right) \rightarrow f_{\infty}(u)$. Since for $n$ large enough we have $\left\|r_{n} u-x_{n}\right\|<\varepsilon r_{n}$ and $r_{n} u \in C_{\infty}$, we get a contradiction with $\left\|f\left(x_{n}\right)-f_{\infty}\left(r_{n} u\right)\right\| \geq \varepsilon\left\|x_{n}\right\|$.

We are now in a position to state and prove the announced criterion.

Proposition 11. Let $K$ be a firm asymptotic cone of a subset $C$ of $X$. Suppose $f$ : $C \rightarrow X$ has a firm asymptotic semi-derivative $f_{\infty}: K \rightarrow X$ which is asymptotically contractive on $K$. Then $f$ is asymptotically contractive on $C$.

Proof. Let us show that relation (1) is satisfied. The observation following Definition 1 shows that we can take $x_{0}=0$ in that definition applied to $f_{\infty}$ and $K$, so that there exists some $c \in(0,1)$ such that

$$
\left\|f_{\infty}(v)\right\| \leq c\|v\|
$$

for $v \in K$ with sufficiently large norm; since $K$ is a cone and $f_{\infty}$ is positively homogeneous, this relation is satisfied for any $v \in K$. Let $c^{\prime} \in(c, 1)$ and let $\varepsilon>0$ be such that $c+3 \varepsilon<c^{\prime}$. Then, taking $\rho>0$ associated with $\varepsilon$ in Definition 8 , for any $x \in C \backslash \rho B_{X}$ we can pick $v \in K$ satisfying $\|x-v\|<\varepsilon\|x\|,\left\|f(x)-f_{\infty}(v)\right\|<\varepsilon\|x\|$, so that we get

$$
\begin{aligned}
\left\|f(x)-f\left(x_{0}\right)\right\| & \leq\left\|f_{\infty}(v)\right\|+\varepsilon\|x\|+\left\|f\left(x_{0}\right)\right\| \\
& \leq c\|v\|+\varepsilon\|x\|+\left\|f\left(x_{0}\right)\right\| \\
& \leq c\|x\|+2 \varepsilon\|x\|+\left\|f\left(x_{0}\right)\right\| \\
& \leq(c+2 \varepsilon)\left\|x-x_{0}\right\|+\left\|f\left(x_{0}\right)\right\|+(c+2 \varepsilon)\left\|x_{0}\right\| \\
& \leq(c+3 \varepsilon)\left\|x-x_{0}\right\| \leq c^{\prime}\left\|x-x_{0}\right\|
\end{aligned}
$$

provided

$$
\varepsilon\left\|x-x_{0}\right\| \geq\left\|f\left(x_{0}\right)\right\|+(c+2 \varepsilon)\left\|x_{0}\right\|,
$$

which occurs when $\|x\| \geq \varepsilon^{-1}\left(\left\|f\left(x_{0}\right)\right\|+(c+3 \varepsilon)\left\|x_{0}\right\|\right)$.

Gathering Corollary 3 and Proposition 11, we get the following result.

Theorem 12. Let $X$ be a uniformly convex Banach space and let $C$ be a closed convex subset of $X$. Let $K$ be a firm asymptotic cone of $C$. Let $f: C \rightarrow C$ be a nonexpansive map which has a firm asymptotic semi-derivative $f_{\infty}: K \rightarrow X$ which is asymptotically contractive on $K$. Then $f$ has a fixed point.

This result does not involve compactness assumptions; such assumptions can be used as criteria ensuring its hypothesis, according to Lemma 7 and Lemma 10. These criteria clarify the links with the results by Luc in [14]. Let us note that the preceding criteria is not as general as the condition of Proposition [2, as the following example shows.

Example. Let $X=\mathbb{R}=C=K$, and let $f$ be the even function given by $f(x)=x$ for $x \in[0,1], f\left(2^{n}\right)=(-1)^{n-1} 2^{n-2}$ for $n \in \mathbb{N} \backslash\{0\}, f$ being affine on $\left[2^{n}, 2^{n+1}\right]$ for $n \in \mathbb{N}$. Then $f$ is nonexpansive and asymptotically contractive, but has no asymptotic semi-derivative, so that Proposition 2 applies, but not the last criterion. 
We will not consider extensions of this result to nonconvex sets $C$ or to more general spaces; see [4], 8]-[10], [16], [24] for example. For application of the criteria using asymptotic compactness, the use of another topology on $X$ would be useful; we do not consider such a question here. We refer to [5], [14], [15], [17]-23] for the use of asymptotic compactness in various fields.

\section{ACKNOWLEDGEMENT}

The author is grateful to an anonymous referee for the suggestion of adding examples and of supplementing Corollary 3 by Proposition 2 .

\section{REFERENCES}

1. A. AGADI and J.-P. PENOT, Asymptotic approximation of sets with application in mathematical programming, preprint, Univ. of Pau, February 1996.

2. Y. BENYAMINI and J. LINDENSTRAUSS, Geometric Nonlinear Functional Analysis, Amer. Math. Soc. Colloquium Publications \#40 Amer. Math. Soc., Providence (2000). MR 2001b:46001

3. F.E. BROWDER, Nonexpansive nonlinear operators in a Banach space, Proc. Nat. Acad. Sci. 54 (1965), 1041-1044. MR 32:4574

4. F.E. BROWDER, Nonlinear operators and nonlinear equations of evolution in Banach spaces, Proc. Symp. Pure Math. vol. 18, Amer. Math. Soc., Providence (1976). MR 53:8982

5. J.-P. DEDIEU, Cône asymptote d'un ensemble non convexe. Application à l'optimisation, C. R. Acad. Sci. Paris 287 (1977), 501-503. MR 56:16320

6. D. GÖHDE, Zum prinzip der kontraktiven abbildung, Math. Nach. 30 (1965), 251-258. MR 32:8129

7. V.I. ISTRATESCU, Fixed point theory, an introduction, Reidel, Dordrecht (1981). MR 83c:54065

8. W. KIRK, A fixed-point theorem for mappings which do not increase distances, Amer. Math. Monthly 72 (1965), 1004-1006. MR 32:6436

9. W.A. KIRK, Nonexpansive mappings and asymptotic regularity, Nonlinear Anal., Theory, Methods, Appl. 40A (2000), 323-332. MR 2001m:47116

10. W.A. KIRK, The fixed point property and mappings which are eventually nonexpansive, in Kartsatos, Athanassios G. (ed.), Theory and applications of nonlinear operators of accretive and monotone type, Lect. Notes Pure Appl. Math. 178, Marcel Dekker, New York (1996), 141-147. MR 97e:47099

11. W.A. KIRK, C. M. YANEZ and S.S. SHIN, Asymptotically nonexpansive mappings, Nonlinear Anal., Theory, Methods, Appl. 33 (1) (1998), 1-12. MR 99g:47128

12. M.A. KRASNOSELSKII, Positive solutions of operator equations, Noordhoff, Groningen (1964). MR 31:6107

13. D. T. LUC, Recession maps and applications, Optimization 27 (1993), 1-15. MR 95e:49021

14. D. T. LUC, Recessively compact sets: uses and properties, Set-Valued Anal. 10 (2002), 15-35.

15. D. T. LUC and J.-P. PENOT, Convergence of asymptotic directions, Trans. Amer. Math. Soc. 353 (2001), 4095-4121. MR 2002e:49028

16. J.-P. PENOT, Fixed point theorems without convexity, Mémoire Soc. Math. de France ${ }^{\circ} 60$ (1977), 129-152. MR 81c:47061

17. J.-P. PENOT, Compact nets, filters and relations, J. Math. Anal. Appl. 93 (1983), 400-417. MR 84h:49032

18. J.-P. PENOT, What is quasiconvex analysis?, Optimization 47 (2000), 35-110. MR 2001a:49024

19. J.-P. PENOT, A metric approach to asymptotic analysis, preprint, Univ. of Pau, June 2001.

20. J.-P. PENOT and C. ZALINESCU, Continuity of usual operations and variational convergences, preprint, Univ. of Pau, 2000 and 2001, to appear in Set-Valued Analysis.

21. B.D. ROUHANI and W.A. KIRK, Asymptotic properties of nonexpansive iterations in reflexive spaces, J. Math. Anal. Appl. 236 (2) (1999), 281-289. MR 2001a:47056

22. S. SINGH, B. WATSON and P. SRIVASTAVA, Fixed point theory and best approximations: the KKM principle, Kluwer (1997). MR 99a:47087 
23. C. ZALINESCU, Recession cones and asymptotically compact sets, J. Optim. Theory Appl., 77 (1993), 209-220. MR 94d:90057

24. E. ZEIDLER, Nonlinear Functional Analysis and its Applications, Part 1: Fixed-Point Theorems, Springer Verlag, New York (1986). MR 87f:47083

Laboratoire de Mathématiques Appliquées, CNRS F.R.E. 2570, Faculté des Sciences, AV. DE L'Université, 64000 PAU, France

E-mail address: jean-paul.penot@univ-pau.fr 